\title{
Anatomic double-bundle ACL reconstruction: a call for indications
}

\author{
Rainer Siebold · Thore Zantop
}

Published online: 23 January 2009

(C) Springer-Verlag 2009

The paper by Kopf et al. entitled "A systematic review of the femoral origin and tibial insertion morphology of the ACL" provides a very well performed literature search looking for measurements of the anterior cruciate ligament (ACL) footprints. The anatomical studies which were analyzed showed a large variability in terms of the size and shape of the ACL anatomy and demonstrate a need for an individual approach to ACL reconstruction.

The anatomy of the ACL and the "double-bundle concept" for ACL reconstruction was one of the most important topics during the last ESSKA meeting in Portugal. One well recognized symposium was entitled "ACL double-bundle, is it really better?" In our opinion, the quintessence of clinical and basic research during the last years might be much better expressed by the question "Anatomic single-bundle or anatomic double-bundle ACL reconstruction; which technique is advantageous for which knee?"

In fact, the primary intention of ACL reconstruction is to regain functional knee stability and normalize the laxity. To achieve this goal, we need to perform a meticulous "insertion site surgery" in order to recreate the highest possible amount of functional insertion site areas on the tibia and femur.

\footnotetext{
R. Siebold ( $\square)$

Center for Knee and Foot Surgery, Sportstraumatology, ATOS Hospital Heidelberg, Bismarckstr. 9-15, 69115 Heidelberg, Germany

e-mail: siebold@atos.de; rainer.siebold@atos.de

T. Zantop

Poliklinik und Klinik für Unfall-, Hand- und

Wiederherstellungschirurgie, Universitätsklinikum Münster,

Waldeyerstr. 1, 48149 Münster, Germany

e-mail: thore.zantop@ukmuenster.de
}

Several clinical studies $[5,6]$ have shown anatomical tunnel placement to be one of the fundamental keys to successful ACL reconstruction. However, the clinical discussion and controversies may also have an important impact on the individual decision for anatomic single- or double-bundle ACL reconstruction.

In the case of small individual ACL insertion sites (length approximately $15 \mathrm{~mm}$ or less) [3], anatomic single-bundle ACL reconstruction may cover most of the insertion site areas on the tibia and femur. These patients may have no significant clinical benefit from an anatomic double-bundle reconstruction and, therefore, a single-bundle technique may be advantageous.

However, in the case of large individual ACL insertion sites (length approximately $15 \mathrm{~mm}$ or more) [3], a singlebundle ACL reconstruction may barely cover parts of the anatomical footprints. In contrast, these patients may have the greatest functional benefits from a doublebundle reconstruction, as the recreated insertion areas are significantly larger compared with a single-bundle ACL reconstruction.

In summary, we think that recent publications in Knee Surgery, Sports Traumatology, Arthroscopy [1, 2, 4], such as the study by Kopf et al., significantly contribute to strategies in anatomical ACL reconstruction. Based on the large individual variability of ACL insertion areas, we should consider different solutions for different patients suffering subjective instability. Depending on the knee, both the single- and double-bundle technique may be valuable options for "anatomical" footprint reconstruction.

Further clinical research may need to address these issues and has to define individual anatomical and functional indications for single- and double-bundle ACL reconstruction. 


\section{References}

1. Colombet P, Robinson J, Jambou S et al (2006) Two-bundle, fourtunnel anterior cruciate ligament reconstruction. Knee Surg Sports Traumatol Arthrosc 14:629-636

2. Edwards A, Bull AM, Amis AA (2008) The attachments of the anteromedial and posterolateral fibre bundles of the anterior cruciate ligament. Part 2: femoral attachment. Knee Surg Sports Traumatol Arthrosc 16:29-36

3. Siebold R, Ellert T, Metz S et al (2008) Tibial insertions of the anteromedial and posterolateral bundles of the anterior cruciate ligament: morphometry, arthroscopic landmarks, and orientation model for bone tunnel placement. Arthroscopy 24:154-161
4. Tállay A, Lim MH, Bartlett J (2008) Anatomical study of the human anterior cruciate ligament stump's tibial insertion footprint. Knee Surg Sports Traumatol Arthrosc. 16:741-746

5. Zantop T, Diermann N, Schumacher T et al (2008) Anatomical and nonanatomical double-bundle anterior cruciate ligament reconstruction: importance of femoral tunnel location on knee kinematics. Am J Sports Med 36:678-685

6. Zavras TD, Race A, Amis AA (2005) The effect of femoral attachment location on anterior cruciate ligament reconstruction: graft tension patterns and restoration of normal anterior-posterior laxity patterns. Knee Surg Sports Traumatol Arthrosc 13:92-100 